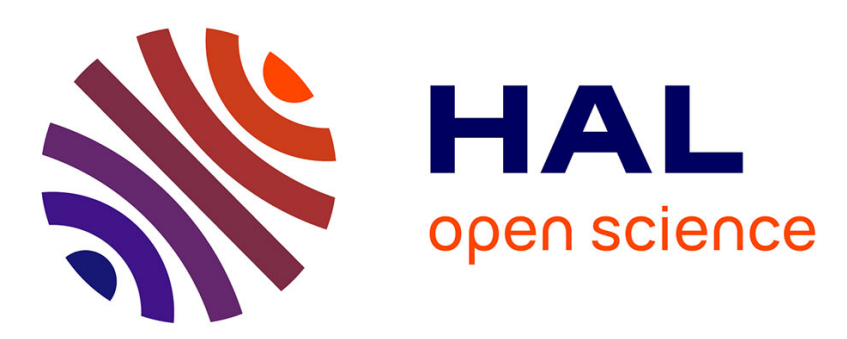

\title{
Les villes et la préférence géographique des affaires en Amérique Latine \\ Anne Collin Delavaud
}

\section{To cite this version:}

Anne Collin Delavaud. Les villes et la préférence géographique des affaires en Amérique Latine. L'Information géographique, 2002, 66, pp.289-309. halshs-00682916

\section{HAL Id: halshs-00682916 \\ https://shs.hal.science/halshs-00682916}

Submitted on 27 Mar 2012

HAL is a multi-disciplinary open access archive for the deposit and dissemination of scientific research documents, whether they are published or not. The documents may come from teaching and research institutions in France or abroad, or from public or private research centers.
L'archive ouverte pluridisciplinaire HAL, est destinée au dépôt et à la diffusion de documents scientifiques de niveau recherche, publiés ou non, émanant des établissements d'enseignement et de recherche français ou étrangers, des laboratoires publics ou privés. 


\title{
Anne Collin Delavaud octobre 2002
}

\section{Les villes et la préférence géographique des affaires en Amérique latine}

\begin{abstract}
Résumé
A côté de Sao Paolo et de Mexico, métropoles reconnues, quelles sont les autres villes attractives pour le monde des affaires en Amérique latine ? La concentration des activités de commandement privilégie certaines capitales, mais n'écarte cependant la possibilité pour des villes plus modestes de capter les entreprises. Cette émergence de nouveaux centres d'affaires témoignent d'une adaptation des territoires urbains et d'une volonté de réaliser des aménagements ou des évènements susceptibles d'attirer des décideurs internationaux.
\end{abstract}

Mots-clefs : Amérique latine, villes, métropoles, entreprises, pouvoir de commandement, aménagement, attractivité.

\begin{abstract}
Besides the town of Sao Paulo and Mexico, well-known metropolis, what other towns are attractive to the world of business in Latin America ? Decision-making activities are mainly concentrated in some capitals, however, it does not deny the possibility, for smaller towns, to arouse the interest of companies. The emergence of new business centres shows an adaptation of urban areas and a will to realise developments or events that might attract international decision-makers.
\end{abstract}

Key-Words: Latin America, town, metropolis, companies, decision-making power, development, attractiveness

Le monde des affaires appartient aux entreprises, mais, aussi aux villes, bases territoriales indispensables à partir desquelles elles pourront diriger leur production, leur commercialisation et leur diffusion d'informations. Les meilleures conditions se trouvent-elles réunies dans les grandes villes de l'Amérique latine, soit 52 agglomérations millionnaires dont quatre de plus de 10 millions d'habitants? Ces villes sont-elles d'emblée mieux placées pour accueillir les nouvelles sociétés ? Celles de taille inférieure ont-elles une place? La compétition entre les villes se fait chaque jour sentir pour que les autorités locales et le secteur privé créent tel ou tel équipement, aménagement ou évènement en faveur de l'attractivité territoriale de la ville, en vue de son insertion à l'économie mondiale. Il ne suffit plus d'offrir un marché, il faut offrir une "valeur ajoutée", un élément de séduction, une qualité des réseaux, voire une esthétique des bâtiments, rendant la ville attractive pour l'investissement et pour les cadres qui y viennent travailler. Il semble qu'une discrimination des grandes villes est même en train de s'effectuer selon leur capacité à séduire les 
investisseurs par les services offerts aux entreprises. Sur quels critères se mesure aujourd'hui l'attractivité des territoires urbains latino-américains ?

La plupart des agglomérations d'Amérique latine sont perçues trop souvent comme des lieux de pauvreté et de vie difficile au point d'omettre leurs atouts. L'énumération des handicaps n'en finit pas : insécurité, pollution, circulation difficile... Marché attractif grâce à leur nombre d'habitants avec une réelle classe moyenne consommatrice, les villes, en rassemblant les trois- quarts des latino-américains, offrent pourtant des atouts déterminants pour les entreprises. La reprise économique du continent au cours de la décennie 1990, a créé une dynamique nouvelle en dépit de violentes crises financières ou politiques qui frappent certains pays avec des répercussions sur le monde des affaires. L' « offre urbaine » de l'Amérique latine est grande, mais la concurrence aussi, obligeant chaque agglomération à présenter une spécificité qui parviendra à capter les médias et à faire reconnaître un atout supplémentaire.

Connaître la perception des chefs d'entreprise sur les villes latino-américaines reste prioritaire. Les investisseurs favorisent la recherche d'une synergie entre cette concentration d'activités et la nécessaire image de marque qu'offre le choix d'une ville. La qualité de leurs quartiers résidentiels et d'affaires est un des éléments déterminants dans les premières implantations des groupes étrangers. L'aspect psychologique et l'image colportée par les médias comptent alors pour beaucoup. Comment les villes parviennent-elles à se faire connaître et à attirer des investisseurs ? La nouvelle attraction de villes, dépassant un million d'habitants, n'est-elle pas significative d'une recherche d'implantation en centres urbains moins importants pour y gagner de meilleures conditions de travail et de vie ce qui relativiserait la réalité de la métropolisation? Les stratégies sont très variées. L'amélioration des équipements et la modernisation des systèmes productifs et de communication permettent aux villes de pénétrer davantage le système international voir mondial.

Il convient dans un premier temps de mettre en évidence les villes décisionnelles au niveau des affaires nationales et internationales à partir de la localisation des sièges puis d'étudier si leur perception par les chefs d'entreprise se rapproche de cette réalité et si de nouvelles implantations se font en faveur d'autres villes. Dans un deuxième temps, il sera montré les efforts des villes pour devenir plus attractives avec des politiques urbaines mêlant marketing urbain, création d'événementiels et consolidation de nouveaux quartiers d'affaires.

Placer la carte numéro 1 : les villes millionnaires de l'Amérique latine

\section{I - La concentration des activités de commandement économique en Amérique latine}




\section{Une « offre urbaine » diversifiée mais dominée par deux métropoles}

Le choix d'une implantation dépend de plusieurs paramètres indiquant la vitalité et l'attractivité des territoires urbains (marché, capacité d'accueil, importance du personnel technique) mais aussi de l'approche culturelle du décideur. Le géographe choisira d'étudier plus spécialement, la position, les réseaux, l'accessibilité, les communications, les transformations territoriales et les aménagements réalisés et à venir pour accueillir les entreprises et leur siège sans oublier les qualités urbaines indispensables pour attirer les cadres et leurs familles. Aujourd'hui, la production, la recherche, la gestion et la commercialisation sont éclatées sur de nombreux sites éparpillés parfois à travers un ou plusieurs continents. Cette réalité au niveau de l'entreprise n'empêche pas le regroupement de certains secteurs en particulier ceux de la recherche et de la direction sur un territoire urbain spécifique ${ }^{1}$. Pour S. Sassen, plus les entreprises se dispersent, plus les centres de décision se concentrent dans des lieux stratégiques que sont les villes mondiales ${ }^{2}$.

Les réseaux actuels de communication et d'information effacent la distance et remettent en question les localisations d'activités. « La plupart des analyses des économistes ou des sociologues sont aspatiales et déconnectées des contraintes » écrit J.P. Paulet, qui ajoute qu'on assiste à un renforcement du pouvoir de certains lieux, de certaines villes car les réseaux exigent des points d'impulsion ${ }^{3}$. L'internationalisation accroît les exportations dans le chiffre d'affaires des entreprises, les investissements directs ou non à l'étranger, le phénomène de multinationalisation des firmes, les migrations internationales, les échanges culturels, les transports, la circulation des informations, les réseaux internationaux de toutes sortes.... ${ }^{4}$.

Capter les capacités des villes à répondre à la mondialisation, c'est-à-dire, l'organisation planétaire de la production et des échanges de biens ou de services, reste un exercice périlleux tant il est difficile de distinguer ce qui est lié à la modernisation, à la croissance des services internationaux et à la montée des réseaux. Les informations sur le sujet sont relativement peu nombreuses et lorsqu'elles existent, elles sont données par la division administrative ne se recoupant pas souvent avec l'agglomération quand elles ne sont pas tout

\footnotetext{
${ }^{1}$ Il ne sera pas étudié les avantages fiscaux et autres proposés par les municipalités et les états.

2 Sassen Saskia, la ville globale, Ed.Descartes, Paris 1996, Cities in a World Economy, Thousand Oaks,CA :

Pine Forge Press ( $2^{\text {nd }}$ ediction, originally published en 1994).

${ }^{3}$ Jean Pierre Paulet, Géographie urbaine. A. Colin, Paris, 2000

${ }^{4}$ Jacques Bonnet, La dynamique des services, Les très grandes concentrations urbaines. SEDES, Paris, 2000.
} 
simplement données par pays ${ }^{5}$. L'analyse comparative reste donc difficile. Les quelques études sur le sujet mettent en évidence les extraordinaires capacités de Sao Paulo et de Mexico, sans qu'il soit possible de réaliser un tableau comparatif de leurs indicateurs communs.

La concurrence est grande sur le plan économique entre les deux agglomérations les plus importantes du continent : Sao Paulo à la tête d'un pays puissant ( 8 rang mondial pour son $\mathrm{PNB}$ ) avec 170 millions d'habitants doit partager avec Rio et Brasilia le pouvoir de commandement tandis que Mexico, avec ses 100 millions de Mexicains entraînés par l'ALENA, catalyse une énorme force, ce qui n'empêche pas la percée de Monterrey.

Le groupe de recherche anglais, Globalization and World Cities Study Group and Network $(\mathrm{GaWC})^{6}$ place Mexico et Sao Paulo au deuxième rang de la classification dans le groupe «Beta», juste après le groupe «Alpha »où se situe Londres, New York, Tokyo et Paris. Cette approche reprend la méthode de S. Sassen, à partir de l'analyse, dans 55 villes, des services de haut niveau, opérant au niveau global dans les services juridiques, financiers, de l'expertise comptable et de la publicité. L'essor de ces trois branches confère aux villes des activités de contrôle et de commandement dans le système international. Il y a trois ans, les deux métropoles latino américaines étaient placées en troisième position dans le groupe « Gamma $»^{7}$.

\section{Carte $n^{\circ} 2$ : Le classement du groupe de recherche sur les villes mondiales}

Les transformations de la dernière décennie sont si fortes qu'il faut, néanmoins, tenter de les observer avec les données disponibles ${ }^{8}$. Une approche descriptive, voire empirique, reposant sur des informations les plus diverses doit permettre de montrer la nouvelle volonté des villes latino-américaines de prendre place dans la compétition internationale.

Tab.1 : Le pouvoir d'achat total de la population par pays et par famille

\begin{tabular}{|l|l|lr|}
\hline & $\begin{array}{l}\text { Pouvoir d'achat de la } \\
\text { population totale * }\end{array}$ & $\begin{array}{l}\text { Pouvoir } \\
\text { /famille/an** }\end{array}$ & d'achat \\
\hline Brésil & 386496 & 8192 & \\
\hline
\end{tabular}

\footnotetext{
${ }^{5}$ Par exemple, les sièges des 500 plus grandes sociétés en Amérique latine sont localisées par pays et non par ville.

${ }^{6}$ L'adresse du GaWC : www.lboro.ac.uk/departements/gy/research/gawc/html

${ }^{7}$ Robert Fossaert, « Les villes mondiales, villes du système mondial », Hérodote, p10 à 25, 2000.

${ }^{8}$ Le palmarès des villes réalisé par la revue Entreprise, en novembre 1999 met en évidence des critères d'attractivité rapportés en fonction du nombre d'habitants : le développement économique (nombre de créations d'entreprises et d'emplois), les capacités d'accueil, la richesse en matière grise et la qualité de la desserte.
} 


\begin{tabular}{|l|l|l|}
\hline Mexico & 383056 & 21720 \\
\hline Argentine $^{\circ}$ & 207967 & 20776 \\
\hline Venezuela & 73730 & 16723 \\
\hline Colombie & 54925 & 8558 \\
\hline Chili & 47190 & 12152 \\
\hline Pérou & 41880 & 10011 \\
\hline Uruguay & 15840 & 16244 \\
\hline Equateur & 8820 & 3582 \\
\hline & & \\
\hline * en millions US\$ & $* *$ en milliers US \$ & \\
\hline
\end{tabular}

Sources: Consultoria Strategy Research Corporation, mai 2000

${ }^{\circ}$ L'information ci-dessus sur l'Argentine ne tient pas compte de la forte dévaluation du peso intervenue depuis.

Il ne s'agit pas d'étudier le rôle de ces villes dans la mondialisation, mais d'identifier les villes attractives pour les affaires à partir de la localisation des centres de décisions des entreprises d'Etat et du secteur privé national et international. Leur place parmi les 500 plus grandes sociétés mondiales appelle à la modestie, elles ne sont que 6, mais il est incontestable que le secteur pétrolier et la banque occupent en Amérique latine une place importante. ${ }^{9}$

Tab. 2 : Les 6 premières sociétés latino-américaines

\begin{tabular}{|l|l|l|l|l|}
\hline & Rang & Revenus & Profit (millions\$) & Siège social \\
\hline Venezuela & & & & \\
\hline PVDSA $^{\circ}$ & 102 & 32648 & 2819 & Caracas \\
\hline Brésil & & & & \\
\hline Banco do Brasil & 246 & 17981 & 465 & Brasilia \\
\hline Petrobras & 280 & 6351 & 970 & Rio \\
\hline Banco Bradesco & 302 & 1564 & 60 & $\begin{array}{l}\text { Osasco } \\
\text { (Sao Paolo) }\end{array}$ \\
\hline Mexique & & & & Mexico \\
\hline Pemex & 144 & 25783 & 1906 & Mexico \\
\hline Telefonos de Mexico & 482 & 10076 & 2628 & \\
\hline & & & & \\
\hline & & & & \\
\hline
\end{tabular}

Sources : 500 plus grandes sociétés mondiales par pays. Fortune, 31 Juillet 2000

○ PVDSA : Pétroleos de Venezuela 
Pour en savoir plus sur le monde des affaires, il faut descendre à l'échelle des entreprises du continent latino-américain et leur implantation. L'ensemble des 250 plus grandes sociétés latino-américaines, activités nationales et internationales confondues, est accessible grâce à la recherche des adresses des sièges sociaux dans l'annuaire des grandes sociétés d'Amérique latine ${ }^{10}$ et grâce à Internet. Classées en fonction de leur chiffre d'affaires, les entreprises d'Etat, souvent consacrées au secteur énergétique, sont parmi les premières et sont souvent implantées dans les capitales (sauf au Brésil). Les activités dominantes sont le pétrole, l'automobile et les télécommunications ${ }^{11}$.

Tab. 3: Localisation des 250 premières entreprises privées et d'Etat en Amérique latine par pays

\begin{tabular}{|lc|}
\hline Mexique & 106 \\
\hline Brésil & 98 \\
\hline Argentine & 24 \\
\hline Chili & 14 \\
\hline Venezuela & 3 \\
\hline Colombie & 2 \\
\hline Pérou & 1 \\
\hline Equateur & 1 \\
\hline Uruguay & 1 \\
\hline
\end{tabular}

Sources : AméricaEconomía, 2 août 2001.

\section{Les implantations privilégiées au Mexique,}

Sur les 250 premières entreprises en Amérique latine, le Mexique regroupe 106 sièges sociaux d'entreprises publiques ou privées, nationales ou internationales. Une étude plus précise sur les 100 premières d'Amérique latine en localise 43 au Mexique, dont 23 dans le District Fédéral, 4 dans l'Etat de Mexico, 1 à Puebla, 9 à Monterrey et dans son Etat, le Nuevo León et 6 sans information sûre.

L'aire métropolitaine de Mexico concentre donc toujours plus de la moitié des sièges sociaux des plus grandes sociétés nationales ou étrangères implantées au Mexique, parmi les plus productives. 4 des 7 entreprises d'automobile, 9 des 14 compagnies du secteur pétrochimique et 5 des 12 sociétés d'équipement électrique $^{12}$. Le choix de la capitale Mexico s'impose facilement comme lieu d'accueil privilégié pour $53 \%$ des entreprises de toute taille, près du pouvoir, mais aussi des consommateurs et des réseaux

\footnotetext{
${ }^{10}$ Major companies of Latin America and the Carribean. 2001, 1500 p, Graham \& Whiteside, London 2001.

${ }^{11}$ Le secteur bancaire n'est pas pris en considération dans cette liste.

${ }^{12}$ Cité par C. Parnreiter, « Mexico City : an emerging city ». El mercado de valores. September- October 2000
} 
Le District Fédéral regroupe, à lui seul, 58,3\% des sociétés à capitaux majoritairement étrangers (84) et abrite encore $52 \%$ des 100 premières sociétés travaillant à l'import-export. Le reste de l'agglomération de Mexico située dans l'Etat de Mexico ne localise que $10 \%$ de ces grandes entreprises nationales.

Il est intéressant de noter une certaine dispersion dans tout le pays avec une présence supérieure dans l'Etat du Nuevo León où Monterrey, capitale de la sidérurgie, joue une certaine attraction, avec 8 des 100 premières entreprises latino-américaines. Sa position géographique et sa forte spécialisation industrielle depuis plus d'un siècle lui assurent une fonction de commandement non négligeable pour une ville qui n'est pas une capitale nationale. Monterrey joue un rôle d'interface avec les Etats-Unis pour le Mexique, comme Miami pour l'ensemble de l'Amérique latine.

En plus, cette présence des sièges sociaux ne se traduit pas par un pourcentage plus élevé d'emplois pour l'aire métropolitaine de Mexico qui connaît une période de désindustrialisation. Il s'agit plutôt d'une nouvelle division du travail avec les villes de la région centrale ou de la frontière septentrionale ${ }^{13}$.

\section{Les implantations privilégiées au niveau du Brésil}

Dans le classement des 250 plus grandes entreprises situées en Amérique latine, la localisation des sièges sociaux des 100 premières indique 35 sociétés brésiliennes dont 25 ont pu être localisées par leur adresse ${ }^{14}$.

Tab. 4: Localisation des sièges sociaux des plus grandes entreprises brésiliennes

\begin{tabular}{|ll|}
\hline Rio & 11 \\
\hline Sao Paulo & 9 \\
\hline Brasilia & 2 \\
\hline Belo Horizonte & 1 \\
\hline Salvador & 1 \\
\hline Porto Alegre & 1 \\
\hline
\end{tabular}

Source : d'après AméricaEconomia, 2 août 2001

Cette approche montre une plus grande dispersion des sièges à travers le pays et le maintien des sièges sociaux à Rio qui reste bien la $2^{\mathrm{e}}$ ville d'affaires du Brésil. La première entreprise brésilienne Petrobras est à Rio, Petrobras District

\footnotetext{
${ }^{13}$ Volkswagen est une exception avec son siège principal à Puebla (au sud-est de Mexico). Ainsi la capitale reste bien la tête de commandement et des relations entre le national et l'international, même si $3000 \mathrm{~km}$ peuvent séparer le siège de l'usine de production.

${ }^{14}$ Recherche sur Internet pour compléter l'information de « Major companies of latin America and Carribean »
} 
également. La capitale fédérale Brasilia, n'a pu, en 40 ans d'existence, centraliser toutes les affaires liées à sa fonction. Pour Sao Paulo, il est intéressant de noter que 3 sociétés ont leur siège dans la périphérie industrielle de l'Aire métropolitaine de Sao Paulo : Sao Caetano, Sao Bernardo et une ville voisine, San Jose do Campos.

Une autre approche basée sur le secteur privé confirme cette organisation en renforçant toutefois le pouvoir de commandement de Sao Paulo et de son Etat. Il faut rappeler que la Bourse de valeurs de Sao Paolo (BOVESPA) est la plus importante de l'Amérique latine, suivie par la Bourse de Mexico.

Tab. 5 : Localisation du siège social des 50 plus grandes entreprises privées au Brésil

\begin{tabular}{|l|l|}
\hline Sao Paulo SP & 18 \\
\hline Rio de Janeiro RJ 16 & \\
\hline Belo Horizonte MG 2 \\
\hline Porto Alegre RS 2 \\
\hline San Bernardo do Campo SP 4 \\
\hline Caetano SP $\quad 2$ \\
\hline Campinas SP 1 \\
\hline Gaspar SC 1 \\
\hline Concordia SC 1 \\
\hline San Joao da Meriti RJ 1 \\
\hline Camaçari BA 1 \\
\hline Osasco SP 1 \\
\hline San Jose do Campos \\
\hline
\end{tabular}

Source: «Les 500 plus grandes entreprises privées du Brésil», Revue EXAME, juin 2000.

\section{Le classement par les chefs d'entreprise des meilleures villes d'Amérique latine pour réaliser des affaires}

Mais, il faut se demander comment ces villes latino-américaines sont perçues. Une enquête réalisée par la revue AméricaEconomía, en 2001 auprès de 500 chefs d'entreprises dans 34 villes latino-américaine et une nord-américaine offre un aperçu de l'attractivité économique de ces villes. Un chef d'entreprise, effectivement, peut donner une opinion valable sur ce qu'il vit au quotidien pour faire marcher son entreprise. Il ne peut méconnaître les réalités locales.

Tab. 7 :Les 20 meilleures villes pour faire des affaires en 2000 


\begin{tabular}{|c|c|c|c|c|}
\hline Pays & Villes & Population & $\begin{array}{l}\text { Pouvoir } \\
\text { d'achat }\end{array}$ & $\begin{array}{c}\text { Classement } \\
\text { de synthèse * }\end{array}$ \\
\hline & & en milliers & $\begin{array}{c}\text { en millions } \\
\text { US \$ }\end{array}$ & \\
\hline USA & Miami & 2,1 & 52223 & 1 \\
\hline Brésil & Sao Paulo & 20 & 96526 & 2 \\
\hline Argentine & Buenos Aires & 12 & 79954 & 3 \\
\hline Chili & Santiago & 6 & 27651 & 4 \\
\hline \begin{tabular}{|l} 
Mexique \\
\end{tabular} & Monterrey & 3 & 19178 & 5 \\
\hline Mexique & Mexico & 20 & 156941 & 6 \\
\hline Brésil & Curitiba & 4 & 2573 & 7 \\
\hline Brésil & Belo Horizonte & 2 , & 11532 & 8 \\
\hline Brésil & Rio de Janeiro & 12 & 31236 & 9 \\
\hline Brésil & Brasilia & 3 & 21566 & 10 \\
\hline Uruguay & Montevideo & 1,4 & 10976 & 11 \\
\hline Mexique & Guadalajara & 3,6 & 22470 & 12 \\
\hline Colombie & Bogota & 7 & 19032 & 13 \\
\hline Brésil & Porto Alegre & 3.8 & 9163 & 14 \\
\hline Costa Rica & San José & 1,4 & 2468 & 15 \\
\hline Argentine & Cordoba & 1,4 & 7555 & 16 \\
\hline Mexique & Puebla & 2 & 6898 & 17 \\
\hline Mexique & Ciudad Juarez & 1,1 & 4883 & 18 \\
\hline Brésil & Salvador & 3 & 5111 & 19 \\
\hline Argentine & Rosario & 1,2 & 6936 & 20 \\
\hline
\end{tabular}

Sources : Strategy Research Corporation 2000 et AméricaEconomía n $^{\circ} 209,31$ mai 2001.

*classement en fonction du marketing stratégique obtenu à partir de nombreux indicateurs sur le coût de la vie, le taux de criminalité, la pénétration d'internet, etc

Des villes de taille très diverses ont été mises en avant, tant par leur nombre d'habitants, d'un à 19 millions d'habitants, que par celui du pays qui les entoure, de 3 à 160 millions habitants ${ }^{15}$. Ce classement a des limites puisque les 500 chefs d'entreprises ne sont pas précisés.

Le classement privilégie des villes "classiques" comme Santiago et des villes "émergentes" comme Monterrey qui dépasse même la capitale Mexico, et de la même façon Curitiba, Guadalajara, Cordoba ; par comparaison avec le tableau

\footnotetext{
${ }^{15}$ Ce classement est limité par le fait que les 500 chefs d'entreprises ne sont pas désignés.
} 
précédent, la ville de Mexico possède une concentration très forte des familles à haut pouvoir d'achat du pays, tandis qu'au Brésil, elles sont réparties dans trois villes (Sao Paulo, Rio de Janeiro et Brasilia).

A la question «Quelles sont les meilleures villes pour faire des affaires ? , les réponses placent une ville des U.S.A. en tête, Miami ${ }^{16}$. Viennent ensuite dans les rangs des gagnantes, cinq autres villes, par ordre d'importance: Sao Paulo, Buenos Aires, Santiago, Monterrey et Ciudad de Mexico.

A une autre question : "Quelles sont les meilleures villes pour attirer les affaires vers le continent latino-américain ?», les réponses confirment Miami, puis Sao Paulo, Ciudad de Mexico, Santiago et Buenos Aires ${ }^{1718}$. Enfin, les chefs d'entreprise constatent tous que le plus grand potentiel d'affaires se trouve en premier à Sao Paulo, Santiago, Buenos Aires puis Ciudad de Mexico. Cette enquête a été réalisée avant les graves crises économiques et financières de fin 2001 en Argentine.

Miami, lieu obligé pour les entreprises de services avec ambitions régionales reste privilégiée par la présence de la plupart des entreprises internationales travaillant avec l'Amérique latine (cf encadré). La Floride tout entière est devenu un interface commode pour tout le continent avec ses liaisons aériennes et maritimes bien développées, plus encore que ne l'est l'Etat de Californie pour le Mexique.

En dehors de Sao Paulo, toujours en tête du peloton des villes latinoaméricaines, 6 autres villes du Brésil appartiennent aux 20 plus attractives du continent. Le repli de Rio, en neuvième position, est récent alors qu'elle a toujours été perçue culturellement par les étrangers comme un lieu d'implantation privilégiée. Toujours jugée comme à vocation politique, Brasilia surprend par sa dixième position. Pouvoir politique et pouvoir économique s'y rapprochent-ils enfin? Le classement d'AméricaEconomia, en introduisant quelques espoirs envers les villes du Nordeste, se base sur des projets qui ne sont pas encore concrétisés.

\section{Carte $n^{\circ} 3$ les 20 meilleures villes pour les affaires}

\section{L'émergence de nouvelles villes attractives}

\footnotetext{
${ }^{16}$ Ch. Girault : «Miami y las nuevas relaciones interamericanas» janv. Mars 1999. Les études du CERI. Fondation Nationale des Sciences Politiques. Paris et d'après Felipe Aldunate Montes : «Yo Quiero Miami ?» AméricaEconomía, août 2000. Panamá.
}

\footnotetext{
${ }^{18}$ La violente crise financière qui a secoué l'Argentine, en décembre 2001, remet en cause l'attractivité de Buenos Aires.
} 
Qu'il s'agisse de petites capitales sur le plan démographique ou de villes qui ne sont pas des capitales, il est intéressant de signaler le maintien de villes dynamiques sur plus d'un siècle comme Monterrey, Puebla, Puerto Alegre, Belo Horizonte, reconnues comme capitales régionales. La percée de villes comme Curitiba ou de San José (Costa Rica) est très significative de leur expérience d'amélioration de la qualité de vie qu'elles ont su faire connaître au reste du monde.

Curitiba est connue dans le monde entier comme la ville la mieux gérée, avec une prise en compte des besoins des habitants. Elle a innové dans le transport collectif, la collecte et le traitement des déchets.

A Recife, la société Porto Digital transforme une partie du vieux port en centre d'exportation de produits informatiques et bénéficie de la spécialisation de l'université locale en informatique. Avec plus de 252 entreprises dans le software en 1999, la réussite de la ville est impressionnante. Mais, en octobre 2001, l'entreprise a choisi de s'implanter à Rio par crainte de son éloignement dans le Nordeste par rapport au centre dynamique du Sudeste.

Le choix de Ford de s'installer dans le Nordeste brésilien à Salvador donne une image industrielle à la ville, complétant sa vocation amorcée, il y a quelques années, par l'implantation de la pétrochimie à Camaçari. 5000 emplois sont prévus par le constructeur automobile. L'implantation de Renault à Curitiba avait démontré, il y a peu, qu'une ville de deux millions d'habitants pouvait réunir toutes les conditions pour le démarrage d'une telle fabrication.

Les réseaux de villes développés autour des deux métropoles Mexico et Sao Paulo ont montré qu'au niveau de la ville moyenne, la concurrence était désormais lancée. Certaines sont devenues des pôles industriels de haute technologie bénéficiant de liaisons rapides avec le centre d'affaires métropolitain proposant de meilleures conditions de vie à ses cadres. Tel est le cas d'Aguascalientes au Mexique $(440000 \mathrm{~h})$ où depuis quelques années s'est implanté l'INEGI (Institut National de Statistiques, de Géographie et d'Informatique) suivi de Rank Xerox, Nissan et Texas Instrument. Au Brésil, c'est la ville de San José Do Campos (500 000 h) qui est devenue la capitale de l'aéronautique et de l'industrie spatiale du Brésil.

A San José (Costa Rica), INTEL a installé en 1997 la plus grande fabrique de microprocesseurs du monde avec un investissement de 300 millions \$US. Cette activité est capable de faire varier le PIB national de $4 \%$ seulement par les seules augmentations et baisses des exportations de ces produits. Par contre, la 
bureaucratie trop lourde a fait partir la société Milicom (USA). La relation villeentreprise n'est pas neutre sur le plan économique.

La montée en puissance des villes de la frontière mexicaine avec les Etats-Unis est aussi significatives : Ciudad Juarez et Tijuana, au 18 et 22e rang en dépit de leur faible pouvoir d'attractivité, se placent néanmoins avant certaines capitales. Une ville de plus de 700000 habitants comme Mexicali, à 150 km de Tijuana, développe ses zones industrielles de façon spectaculaire en accueillant de gigantesques établissements de production des multinationales comme Daewoo, Sony ou Mitsubishi pour devenir la capitale électronique de la frontière et de l'ALENA. Ses atouts (des espaces disponibles, de l'eau en quantité et une solide politique de promotion) ont brutalement été frappés par la récession américaine.

Les capitales Lima, Caracas, Asunción et la Paz n'ont pas actuellement une image de marque valorisante: trop de misère, trop de crises politiques, trop d'isolement sans doute.

\section{La préférence géographique des affaires en Amérique latine}

Le classement établi par la revue AméricaEconomía repose sur une synthèse de divers critères mettant en évidence l'attraction exercée par certaines villes en fonction de l'importance des nouvelles technologies, des foires, de leur offre commerciale, de la rapidité de circulation dans les villes, d'un bon niveau de sécurité.

Les villes sont classées en fonction de la qualité de leur image de marque. Il s'agit à la fois de la capacité de séduction de la ville et des efforts de marketing mise en place par les autorités qui en font une règle de stratégie primordiale pour gagner la confiance des investisseurs.

\section{LA PRESENTATION DU TABLEAU 8 DOIT ETRE PLUS RÉDUITE}

Tab. 8 : Les villes les plus attractives d'Amérique latine selon l'enquête de la revue AméricaEconomía

\begin{tabular}{|l|}
\hline Villes très attractives \\
\hline Miami \\
\hline Buenos Aires \\
\hline Curitiba \\
\hline Recife \\
\hline Villes attractives \\
\hline Santiago \\
\hline Rio de Janeiro \\
\hline
\end{tabular}




\begin{tabular}{|l|}
\hline Bogotá \\
\hline San José \\
\hline Salvador \\
\hline San Salvador \\
\hline Fortaleza \\
\hline Villes moyennement attractives \\
\hline Sao Paulo \\
\hline Monterrey \\
\hline Cd de Mexico \\
\hline Guadalajara \\
\hline Cordoba \\
\hline Puebla \\
\hline Cd de Panamá \\
\hline Lima \\
\hline León \\
\hline Són :
\end{tabular}

Sources : AméricaEconomía n²09, 31 mai 2001

Les autres villes qui ne sont pas citées exercent une image de marque faible, certaines seraient même négatives comme Caracas, Belo Horizonte, Guayaquil et La Paz.

Cette enquête a ses limites. La prise de position est très subjective même si elle a été pondérée par des critères précis (criminalité, attaques, difficulté de circulation). La pollution ou encore la bureaucratie qui frappent dramatiquement la majorité des villes n'ont pas été retenues. Les dirigeants, selon l'enquête, refuseraient, s'ils avaient vraiment le choix, de vivre à Bogota, Caracas, Lima et Sao Paulo. Ils le font pourtant. On ne souhaite pas vivre à Sao Paulo, mais on veut y travailler et l'on y réside par force. L'essor des affaires est plus fort à Bogotà qu'à Medellin ou à Cali. La guerre civile et les systèmes mafieux qui coûtent la vie ou le déplacement à des milliers de personnes n'ont pas mis un frein à la pénétration des entreprises internationales ${ }^{19}$. «Le kidnapping des cadres augmente de $15 \%$ tous les ans (3000 enlèvements l'an dernier). La Colombie, le Mexique et le Costa Rica figurent en tête des pays les plus dangereux et les plus chers pour les rançons $»^{20}$.

Curieusement, Montevideo située en $11^{\mathrm{e}}$ position pour les affaires, n'a qu'un faible pouvoir d'attraction par rapport à des villes à réputation difficile. Or, qui a vécu à Montevideo ne peut vivre ensuite ailleurs avec le même sentiment de sécurité et bénéficie d'un cadre de vie aussi achevé. Tracasseries administratives

\footnotetext{
${ }^{19}$ Un « savoir-vivre » en Colombie est indispensable puisque des morceaux de territoire et des quartiers sont à éviter. En respectant certaines mesures, il est possible de faire des affaires en Colombie ,affirment les chefs d'entreprise français présents au Colloque du CFCE du 11 décembre 2001, à Paris.

20 Valeurs Actuelles du 26 avril 2002.
} 
et faiblesse du marché en dépit d'un bon niveau de vie n'ont pas favorisé l'implantation d'entreprises pour profiter de la proximité du Brésil et de l'Argentine.

Après avoir étudié la relation entre la localisation des affaires à partir des sièges sociaux et la réalité subjective des choix d'implantation, force est de constater que certaines villes offrent une image négative sur le plan résidentiel alors qu'elles sont attractives sur le plan économiques.

L'attractivité territoriale favorise en général les métropoles qui ont su valoriser leur image de marque. Les retombées des choix stratégiques du marketing urbain sont longues à percevoir. Une nouvelle création d'entreprise, comme celle de Ford ou de Renault, offrant des centaines d'emplois directs reste exceptionnelle et demande des années de préparation dans l'évaluation des risques et le montage financier. Toute cette interaction entre les acteurs institutionnels et les acteurs économiques favorise des politiques urbaines expérimentant des changements de rôles intervenus dans la construction récente de la ville à la suite d'un certain retrait de l'Etat en Amérique latine. Les acteurs sociaux ont aussi une capacité d'intervention peut être plus forte qu'auparavant grâce aux médias locaux et internationaux. L'arrivée de nouvelles entreprises du secteur privatisé souvent associées aux capitaux étrangers sert de balancier dans ce jeu de pouvoir à quatre. Il est clairement admis aujourd'hui que la municipalité ne joue plus seulement le rôle d'entité régulatrice avec les avantages offerts, mais aussi celui de promotrice de l'action privée. Des prêts de la BID se font sur cette orientation. D'autres facteurs clés de l'attractivité territoriale sont à prendre en considération : fiscalité, restauration de la sécurité, qualité de l'environnement, efficacité des services de santé et d'enseignement, qualité des infrastructures et des transports publics, beauté des espaces...

\section{II - L'adaptation des territoires urbains aux affaires}

Devenus des acteurs de la scène internationale, les villes se sont transformées pour répondre à leur nouvelle vocation. Des dynamiques de quartiers recomposent aujourd'hui l'espace urbain, mettant en évidence des quartiers spécialisés et mieux desservis, identifiés souvent par un élément architectural phare qui dominera l'immense territoire urbain qui s'étale sur des dizaines de $\mathrm{km} 2$. Si la ville sert de moteur au développement économique national et international, ces quartiers favorisés entraîneront par leurs activités des effets sur l'ensemble des quartiers populaires.

\section{La réalisation d'édifices de prestige et d'aménagements reconnus}


Le regard porté sur la ville et plus particulièrement le centre-ville a favorisé des aménagements urbains et des créations d'équipements souvent fort prestigieux marquant la volonté d'entrer dans le circuit des villes mondiales. Si ces éléments de valeur ajoutée sont utiles, tant mieux, s'ils se voient facilement, c'est encore mieux. Leur coût démesuré est à la hauteur de l'ambition de la ville, voire de l'élu principal.

Mexico, après la construction du superbe édifice emblématique de la Bourse, s'offre la Torre Mayor, le gratte-ciel le plus haut d'Amérique latine ${ }^{21}$. Le nombre de Word Trade Centers construits dans chacune des grandes villes est bien un signe de modernité en direction des entrepreneurs. Egalement, la rénovation des aéroports fait partie des obligations des cités candidates à l'international. San José a modernisé son aéroport en 2001 pour recevoir 6 millions voyageurs. Le nouvel édifice de l'aéroport international de Rio offre une très belle architecture tandis que celui de Sao Paulo (Congonhas) est désormais conçu comme un centre de services avec des opérateurs de tourisme, des loueurs de véhicules, des banques et autres facilités pour les voyageurs, les hommes d'affaires ou les touristes. ${ }^{22}$

Depuis plusieurs années, d'énormes efforts ont contribué à changer le paysage et les activités des centres historiques. Les autorités locales ont été confrontées à la prise en compte du patrimoine qui fait l'objet d'un encadrement législatif souvent ancien même s'il n'est pas toujours respecté. La redécouverte du passé s'inscrit à la fois dans une recherche d'identité et une marche vers une modernité autonome. Le patrimoine n'est pas un héritage, c'est la construction permanente d'un passé et c'est précisément cette construction qui permet d'intégrer un certain nombre de situations contemporaines.

Après une phase de sauvetage des monuments du patrimoine, une action en faveur des équipements de base et de la circulation a permis de revaloriser le centre sur le plan des activités commerciales. Ce nouveau dynamisme territorial $^{23}$ n'a pas réglé les problèmes de logement des habitants souvent pauvres qui occupent ces vieux édifices taudifiés, mais des efforts ont progressivement redonné au moins une façade en attendant mieux.

Les exemples de réhabilitation sont nombreux en Amérique latine et ont fait preuve, en général, de beaucoup de goût, de savoir-faire et d'attention à la vie du

\footnotetext{
${ }^{21}$ Réalisé par un groupe mexicain (ICA) et canadien (Reichmann), il atteindra, en 2003, 55 étages et 285 mètres de haut. Ce projet de 230 millions \$ dépassera la tour Pemex également à Mexico 214 m, la Tour des Ministères à Caracas $215 \mathrm{~m}$ ou la tour Mirage, à Panamá (172 m).

22 Pour la capacité hotelière, Sao Paulo dépasse largement les autres grandes villes avec ses 60000 chambres d'hôtels (New York : 90 000).

${ }^{23}$ SIRCHAL. Ministère des Affaires étrangères. Paris. WWW.archi.fr/sirchal
} 
quartier. A Buenos Aires, la récupération des quais et des bâtiments de stockage du port désaffecté (Puerto Madero) a créé un ensemble urbain de luxe séparé seulement par une grande avenue du centre d'affaires historiques. La réussite a été longue à venir, mais s'y trouvent, aujourd'hui, un hôtel 5 étoiles, des bureaux sophistiqués d'agences, des restaurants réputés et une université privée ${ }^{24}$. La ville a gagné une zone centrale nouvelle prestigieuse en bordure des quais. Les investisseurs ont été payés de leurs efforts. L'agence Corporación Madero vend aujourd'hui ses conseils à de nombreuses villes (à Santos et même à Valparaiso).

A Santiago, la réhabilitation de la station de chemin de fer Mapocho en centre culturel permet d'accueillir des réunions internationales. Une ancienne prison est transformée en édifice intelligent pour bureaux et les rives du torrent Mapocho sont devenues une " coulée verte".

Même Montevideo ${ }^{25}$ a fini par réhabiliter son quartier du port, notamment les édifices jouxtant la Ciudad Vieja et les bâtiments desservant entre autres la liaison maritime par aéroglisseur avec la capitale de Buenos Aires. Plus encore, on restaure la gare pour en faire un centre culturel et commercial et l'on construit une tour résolument high-tech (Torre Fenix) . Ce front d'estuaire exceptionnel où alternent sur plusieurs $\mathrm{km}$ des plages et des petits ports de plaisance offre sans conteste une qualité de vie : en moins de 15 minutes on peut se rendre à son bureau, au lycée, au centre commercial ou à la plage.

Dans le domaine de l'aménagement intégral ${ }^{26}$, Quito offre l'exemple d'une réussite d'adaptation de son centre historique aux réalités économiques locales. En plus des mesures de sauvetage et de restauration de son patrimoine colonial, magnifiquement préservé à travers les siècles, les autorités municipales ont, grâce à un prêt de la $\mathrm{BID}^{27}$, travaillé à la fois la revitalisation économique et la question difficile des maisons anciennes taudifiées (parkings, tramway, hôtels) au point que le tourisme, l'artisanat et le commerce apportent de véritables valeurs à ce quartier ${ }^{28}$.

Les aménagements de réhabilitation devenus courants dans la plupart des villes expriment la volonté des décideurs de réhabiliter l'image de marque de leur métropole et de favoriser les activités économiques génératrices de ressources pour tous.

\footnotetext{
${ }^{24}$ Des 2500 millions investis dans Puerto Madero, $70 \%$ sont d'origine privée.

${ }^{25}$ Jean Claude Herschon, « la vieille ville de Montevideo, enjeux et contradictions d'un espace paradoxal ». L'ordinaire latino-américaniste. $\mathrm{n}^{\circ} 182$, octobre 2000.

${ }^{26}$ Anne Collin Delavaud, "De nouvelles dynamiques urbaines au coeur des Andes. Aménagement et émergence d'une métropole : Quito (Equateur ». Revue de Géographie de Lyon, vol. 74/4 1999.

${ }^{27}$ Grâce, entre autre, à un prêt de la BID de 41000 \$ complétant l'apport équatorien de 10000 \$

${ }^{28}$ Une expérience réussie, le relogement sur place associé à la réhabilitation des édifices taudifiés grâce à la méthode Pact-Arim, une association française spécialisée dans les quartiers anciens et qui intervient depuis l'expérience de Quito dans plusieurs villes d'Amérique latine.
} 


\section{L'émergence de quartiers d'affaires et la recomposition territoriale}

Depuis une dizaine d'années, on assiste à une nouvelle dynamique spatiale avec le renforcement de certaines spécialisations fonctionnelles impliquant la création d'espaces urbains. La réhabilitation de certains secteurs anciens au profit non seulement des activités de service renouvelle aussi la composition territoriale des décennies précédentes.

Le classique glissement depuis le centre historique vers un quartier voisin traversé par une ou plusieurs grandes avenues est désormais insuffisant pour répondre aux nouvelles demandes. On assiste à une explosion d'architecture pour mettre en valeur les secteurs financiers, informatiques et touristiques sur la base de concours internationaux sur projet. Ceux-ci sont de plus chargés de restituer la culture locale dans les réseaux de la mondialisation. La délocalisation des sièges bancaires est significative de cet accompagnement des affaires. Le centre traditionnel a perdu une partie de sa fonction au profit des autres axes.

A Sao Paolo l'essor est si brutal que périodiquement le quartier d'affaires devient insuffisant et exige une création de nouveaux espaces porteurs des mêmes centralités ${ }^{29}$. Jusqu'en 1940, le centre-ville historique autour de Praça da Se a accueilli dans ses premiers gratte ciel les banques et les sièges sociaux de l'économie pauliste issue du café, de la construction et des premières entreprises. Entre 1940 et 1960, les activités se sont étendues sur le quartier voisin (centre nouveau) autour de la place de la République. A partir de 1960, l'avenue Paulista, quartier aisé créé au début du siècle, devient le secteur des banques et des sièges sociaux. Située à $4 \mathrm{~km}$ du centre historique, cette avenue offre très vite des activités culturelles de premier plan grâce à la construction d'édifices spécialisés glissés au milieu des tours. Dès 1980, l'architecture donne une image aux sociétés qu'elle abrite. Les encombrements des centres précédents et le besoin sans cesse grandissant de nouveaux bureaux adaptés ont fait migrer des sièges sociaux vers l'avenue Fara Lima, axe situé à environ $4 \mathrm{~km}$ de la précédente. Aujourd'hui, tout le secteur Berrini, au-delà du río Pinheiros, se couvre de tours high-tech pour abriter les firmes internationales qui ont trouvé là des terrains bon marché et une accessibilité plus aisée. Ces tours isolées les unes des autres n'ont pas encore créé un tissu urbain de services. Les autres axes ne sont pas abandonnés pour autant. Aujourd'hui, les activités s'installent dans le prolongement de l'avenue Farah Lima. 
A Mexico, les équipements de centralité se sont échelonnés, dès 1970, tout au long du Paseo de la Reforma, de l'avenue Insurgentes Sur, ainsi que dans la colonia Polanco vers l'Ouest. Ces deux grands axes se renforcèrent à leur extrémité, avec la création d'ensembles commerciaux et de bureaux, à Périsur et à Santa Fé. Le centre traditionnel de bureaux jouxtant le centre historique a largement souffert du séisme de 1985 puisque 15 ans après, la zone (Alameda) est restée désaffectée malgré sa position et la modernisation des équipements voisins. La fameuse Torre Latina des années 50 est en cours de recyclage. C'est le long de ces axes que "se développe depuis quelques années une stratégie d'image qui donne un rôle essentiel à l'architecture $» .{ }^{30}$ L'architecture de la Bourse de valeurs est emblématique de l'image de pôle financier que Mexico veut donner au reste du monde. Aux Bosques de las Lomas, on assiste à une compétition entre les entreprises «qui se donnent à voir». Comme ailleurs, l'architecture mexicaine met en valeur tantôt les influences high-tech et tantôt celles de son passé indien, parfois, elle réussit à synthétiser les deux.

Le District Fédéral, avec ses différents quartiers d'affaires, concentre toujours l'essentiel des activités de commandement avec les deux tiers du PIB et les trois quarts du PIB des services. Les bureaux se dispersent dans les cinq délégations : Alvaro Obregon, Miguel Hidalgo, Benito Juarez, Cuauhtémoc et Coyoacán. On assiste, comme dans les autres villes latino-américaines, à des créations de quartiers de bureaux et d'activités spécialisées dans les services autour de nouveaux centres commerciaux ou des Word Trade Centers localisés dans la première couronne urbaine, à moins que la zone péri-centrale n'offre des espaces suffisants pour réaliser ces infrastructures. Au-delà du District Fédéral, les districts de l'Etat de Mexico créent de nouvelles zones industrielles regroupant les activités de production.

Cette évolution existe aussi dans les autres métropoles et une des conséquences immédiates apparaît être le renchérissement des prix du foncier et de l'immobilier selon cette côte psychologique qui impose pour tel ou tel quartier d'implantation des activités ou de la fonction résidentielle. A moyen terme, c'est le coût de la vie qui subit une augmentation irrationnelle. Le dernier rapport de The Economist Intelligence Unit place Buenos Aires pour la seconde fois consécutive, comme la ville la plus chère de la région et une des plus chères du monde, seulement derrière Tokyo et Londres. Mexico est entrée aussi dans le club des métropoles aux coûts de vie élevés dépassant de peu Paris. Vient ensuite Caracas. Le rapport note de légères augmentations à Sao Paulo et à Rio et même à Quito.

\footnotetext{
${ }^{30}$ F. Tomas, O.Terrazas, « Mexico : une ville plus humaine pour cette fin de siècle ». Revue de Géographie de Lyon, vol 74 /4, 1999.
} 


\section{Se faire connaître par des événementiels internationaux}

Aujourd'hui, pour se faire connaître, il faut proposer une idée autour d'une rencontre s'adressant aussi bien aux pays du Nord que ceux du Sud. Mexico joue à fond la carte du tourisme et de la culture avec les expositions internationales de son Musée d'Anthropologie, la restructuration de l'Auditorium et la création d'un Centre National de la Culture et des Arts.

Depuis plusieurs années, Sao Paulo est à l'avant-garde des mouvements artistiques grâce à ses nombreux musées dont le MASP et à ses événements artistiques comme la Biennale d'Art qui, depuis 1951, est l'une des plus importantes manifestations artistiques du monde. La "Muestra del Redescubrimento" a accueilli 1,8 million de visiteurs en six mois lors de l'exposition de 1992. Rio, toujours consacrée capitale culturelle du Brésil malgré la place prépondérante de Sao Paulo, n'est plus la seule à offrir des évènements qui attirent du monde entier. Toutefois, la ville a été choisie par la Fondation R. Guggenheim pour la création d'un musée de la même envergure que celui de Bilbao. Il sera localisé dans la zone portuaire, près de la Place Mauá, exprimant par sa localisation un renouveau politique en faveur du centre ancien $^{31}$.

Profitant de nombreux avantages fiscaux, le secteur privé brésilien investit à Sao Paulo dans des infrastructures culturelles et défend des créations que la puissance publique n'est pas en mesure d'assumer, ce qui a permis à la métropole de devenir en une dizaine d'années, la capitale culturelle du cône sud. Le SECS (Service Social du Commerce) organisme privé joue un rôle capital dans ce rayonnement. Il est le plus gros opérateur culturel du Brésil avec un budget dépassant largement celui du ministère de la culture brésilien. D'immenses salles de spectacle ou d'exposition, parfois dans d'anciennes usines réhabilitées, s'ouvrent dans les différents quartiers de la ville pour accueillir des programmes internationaux.

Cette démarche événementielle a déjà quelques années puisque Rio était devenue la capitale de l'environnement grâce à son forum mondial en 1992. Il semblerait que les grandes villes cherchent à se démarquer autour d'un thème favorisant la sympathie du public international presqu'autant que celle d'une spécialisation d'activités. Santiago, siège de l'assemblée annuelle de la BID, souhaite s'affirmer comme capitale financière de l'Amérique latine. Dans un

\footnotetext{
${ }^{31}$ Après Bilbao, Berlin, Venise puis Las Vegas, la fondation s'installe pour la première fois en Amérique latine. Outre Rio, il est prévu des études de faisabilité pour réaliser deux musées plus petits à Salvador, Recife ou Curitiba, soit un investissement total de 200 à 500 millions \$US.
} 
secteur tout autre, le premier Forum Social Mondial, en janvier 2001, suivi d'un deuxième en 2002 à Porto Alegre, a lancé cette ville du sud du Brésil sur une rampe étonnante en faisant d'elle pendant quelques jours le lieu de rencontre des antimondialistes...

Dans le domaine des congrès internationaux, Rio était, il y a quelques années, au 21 e rang avec 68 congrès puis, Buenos Aires au 26e rang avec 51 congrès et La Havane au $30^{\mathrm{e}}$ avec 46. Sao Paulo et Mexico sont absentes de ce classement sans doute pour leur réputation d'insécurité. Il faut rappeler que Miami a misé sur l'ensemble de l'Amérique (nord et sud) pour son festival de mode avec son "Fashion week of the Americas" dans l'espoir de jouer un rôle dans ce secteur comme Paris, Milan ou New York.

Sao Paulo est, incontestablement, reconnu pour être la première ville de foires, sans compter les dépenses des 4 millions de visiteurs qui séjournent quelques jours à cette occasion dans la ville ${ }^{32}$. Néanmoins, Buenos Aires recevait plus de 66 foires par an contre 39 à Sao Paulo, 29 à Mexico et 45 à Monterrey. Santiago, avec 25 foires, possède une des plus importantes manifestations aéronautiques : la Feria Internacional del Aire y Aeronáutica (Fidae).

Les villes du Nordeste brésilien (Fortaleza, Recife, Salvador) jouent à fond la carte du développement touristique grâce à la qualité de leurs plages, à la main d'œuvre bon marché, à leurs efforts de promotion ${ }^{33}$. Des vols directs leur assurent depuis peu des liaisons plus commodes avec l'Europe et les Etats-Unis.

\section{Les géants de la communication sont à Mexico et à Rio}

Le monde latino-américain et espagnol est le deuxième marché audiovisuel au monde avec une couverture se situant entre 85 et $90 \%$ des foyers. Le développement du câble y est spectaculaire. Les principales chaînes de télévision sont très internationalisées, exportant les fameuses telenovelas (feuilletons), mais surtout en s'attaquant aussi au marché de l'information. Cette internationalisation se traduit également par une participation dans le capital de plusieurs chaînes de télévision étrangères. Le Mexique emboîte le pas sur le modèle américain, les autres pays latino-américains ne devant suivre que plusieurs années après.

\section{POUR ALLEGER CETTE PARTIE SANS TABLEAU JE PROPOSE UN ENCADRE}

\footnotetext{
${ }^{32}$ Buenos Aires attire seulement 670000 visiteurs. Sa foire agricole a une réputation mondiale.

${ }^{33}$ Le Nordeste bénéficie d'un certain retrait de Rio dans cette primauté touristique en dépit d'efforts exceptionnels pour sécuriser la plage de Copacabana.
} 
Titre Deux métropoles dominent la communication du continent latinoaméricain

Deux sociétés dominent le marché mexicain : Televisa créée en 1932 est le plus grand groupe de médias du monde hispanophone avec un chiffre d'affaires de 1,68 millions de \$ et Tv Azteca (450 millions \$). Avec 4 chaînes de télévision, Televisa truste $80 \%$ de l'audience-télévision du pays (100 millions d'habitants). C'est le premier catalogue de programmes au monde et le premier fabriquant de feuilletons 1000 heures par an traduites en 32 langues et diffusés dans 114 pays. La société compte 6 stations de radios, un pôle d'édition avec 30 millions de disques vendus par an, mais aussi 3 équipes de foot, avec la gestion du stade azteca qui emploie 17000 salariés.

Au Brésil, TV Globo est le deuxième empire latino-américain de la télévision et de la radio avec 9 chaînes de télévision, des complexes de studio de Projac sur 130 hectares près de Rio, avec Globo International, une société de distribution et de programmation, Radio Globo et ses 18 stations dans le centre et le sud du pays. C'est aussi la musique avec la société Sigla présente sur $22 \%$ du marché et la presse avec le quotidien $\mathrm{O}$ Globo, ainsi que de très nombreux magazines. Editora Globo est le numéro 2 de l'édition en Amérique latine. Le groupe intervient également dans les secteurs culturel, financier, immobilier et même agroalimentaire.

Dans le domaine de la communication téléphonique, le Mexique et en priorité sa capitale occupent la 16e place (sur 25 étudiées) avant Zurich ou Singapour, grâce aux efforts réalisés au cours de la dernière décennie. Avec la privatisation, en 1990, de Teléfonos de México (Telmex) puis la formation d'un consortium Southwestern Bell et France Télecom. Ce dernier et le Groupe Carso sont de dimension mondiale. Ils contrôlent Telmex et assurent au pays une infrastructure puissante avec une transmission à haut débit. Les lignes de téléphone ont doublé entre 1990 et 1999. Elles sont presque toutes numérisées contre seulement $29 \%$ en 1990. Ces progrès techniques massifs ont facilité les communications avec le réseau nord américain, et ont multiplié le nombre des usagers du téléphone ainsi que les utilisateurs d'internet. Un tiers des appels internationaux réalisés au Mexique vient de la capitale.

La géographie des réseaux de fibre optique comme celle des internautes suit rigoureusement les quartiers privilégiés, mais elle évolue vite. Un réseau sousmarin de fibres optiques doit entourer le continent. Déjà, les villes de Sao Paulo, Rio, Buenos Aires et Santiago sont reliées. Les grandes villes latino américaines ont donc tous les outils nécessaires pour les liaisons internes et externes ${ }^{34}$.

\footnotetext{
${ }^{34}$ Le développement du câble avait fait de l'Argentine le deuxième pays câblé après les Etats-Unis.
} 


\section{Conclusion}

Cette étude a tenté de montrer les villes émergentes du continent prêtes, à la fois, à répondre aux besoins nationaux et à la dynamique mondiale. Elle a voulu insister sur le fait que les métropoles d'Amérique latine peuvent prétendre à être des centres décisionnels autrement que dans la dépendance au monde développé. Il est encore difficile de prouver que ces agglomérations ne sont pas que des territoires éclatés, à problèmes, mais qu'il existe une dynamique économique avec une interaction très forte des secteurs formel et informel, qui n'a pas échappé aux investissements étrangers.

Certaines villes latino-américaines ont donc acquis les caractéristiques propres aux villes mondiales tout en gardant des caractères périphériques. Ce sont bien des points d'impulsion qui exercent effectivement en Amérique latine une fonction importante de relais avec le reste du monde. Mais, plus que cela, cette capacité globale indubitablement donne les moyens de diriger et de transformer dans la nation concernée mais aussi dans les pays voisins. La localisation des investissements étrangers et la création de bureaux régionaux chargés de plusieurs pays en sont la preuve. Plus que jamais, ces agglomérations jouent le rôle d'interface entre les circuits économiques mondiaux et les espaces régionaux.

Deux métropoles, Mexico et Sao Paulo, sont, de très loin, les premières métropoles d'Amérique latine, presque prêtes à entrer dans le club très limité des villes mondiales. D'autres en sont encore éloignés tout en devenant peu à peu « des nœuds d'entrecroisement de flux mondialisés ${ }^{35}$ dont profite le réseau de villes qui les entourent tandis que leur arrière-pays y échappe le plus souvent. Face à la métropolisation renforcée cette dernière décennie, des agglomérations moyennes apparaissent comme des lieux d'accueil tout à fait satisfaisants, grâce à l'accessibilité facilitée par le développement aérien et par la télématique, pour répondre à la forte demande en qualité de vie. Pourtant, les métropoles latinoaméricaines se trouvent confrontées à des défis sans cesse plus grands et plus complexes. Outre, une meilleure gestion urbaine, plus démocratique, les villes doivent régler leurs problèmes hérités d'un passé proche où le laisser-faire l'emportait, pour permettre à des millions de citadins de trouver leur place dans les immenses quartiers émergents. Moteurs du développement économique sous influence mondiale, elles doivent évidemment se transformer comme les entreprises elles mêmes, pour répondre aux nouveaux processus de production, d'échange et de concurrence.

\footnotetext{
${ }^{35}$ P. Veltz, «L'archipel des villes et des entreprises ». Villes en développement , n 40. Juin 1998.
} 


\section{LES DEUX ENCADRES SONT A SUPPRIMER}

S'il reste un espace, mettre celui qui convient.. ;

\section{La place exceptionnelle de Miami dans les affaires latino-américaines}

A la tête de l'Etat de Floride (USA) comptant 15 millions d'habitants dont le produit équivalent à celui du Brésil, Miami est une agglomération de 4 millions habitants qui a su magnifiquement jouer, depuis deux décennies, son rôle d'interface entre le nord et le sud, de tête de pont commercial entre l'Europe, les Etats-Unis, l'Amérique centrale, les Caraïbes et des relations non négligeables avec le sud du continent. Seul Mexico échappe à cette relation car elle a un accès direct vers les Etats-Unis grâce à sa frontière terrestre du nord.

Le potentiel économique d'une ville n'est plus le seul point décisif pour les décideurs, d'autres facteurs entrent en compte. Une enquête d'AméricaEconomía publiée en août 2000 désigne Miami, comme la ville la mieux placée, pour la relation potentielle d'affaires et coût, tout en offrant un bon niveau d'éducation, une culture latine dominante (touristes et résidents) et, enfin, une qualité de vie internationalement enviée. Ces qualités ont fait d'elle le siège latino-américain d'innombrables multinationales. Si elle n'est pas un grand centre financier, les transactions se font à distance. La plupart des cabinets d'affaires des villes d'Amérique latine y ont un compte bancaire pour traiter des affaires internationales.

Les deux outils de cette fonction commerciale sont, pour Christian Girault, le port et l'aéroport dont les activités sont essentiellement internationales : hub et plate-forme multimodale avec des échanges tant de marchandises que de passagers. L'essor du tourisme, et en particulier, le boom des croisières, est décisif renforçant la fonction résidentielle et commerciale. C'est le point d'accueil «obligé » des élites « latino » qui viennent y faire leurs achats, et y utiliser un grand nombre de services bancaires et de santé. Mexico reste un lieu privilégié de réception des capitaux latino-américains privés. Cet essor lié à l'histoire cubaine des dernières décennies a modifié la composition ethnique de la ville au profit des " Latinos" qui représentent aujourd'hui $45 \%$ du total. Miami est devenue une véritable ville multiculturelle. "Capitale nordique de l'Amérique latine » elle a réussi par sa dynamique a attiré les divisions latinoaméricaines de grandes sociétés multinationales (IBM, Microsoft, Federal Express, Motorola, Volfswagen, Telefónica, United Air Lines et Lucent Technologies). Sans doute la comparaison avec Singapour permet de resituer le 
choix de Miami comme une localisation neutre permettant de mieux planifier les stratégies à long terme de la région et d'éviter ainsi les crises économiques et les politiques des pays du Sud. Toutefois, la distance avec Sao Paulo, Mexico ou Buenos Aires faire perdre la connaissance de la réactivité des consommateurs en dépit des efforts en faveur des télécommunications. Les universités doivent élever encore leur niveau pour convaincre.

6 des 11 plus grandes sociétés de service aux producteurs ont leur bureau régional à Miami significatif du rôle de centre pour l'Amérique latine prenant donc une partie du rôle qui aurait dû revenir à Mexico. Début 1990, un tiers du volume commercial entre les Etats-Unis et l'Amérique latine passait par Miami. $\mathrm{Si}$ on ne tient compte que de l'Amérique centrale et des Caraïbes, la participation de Miami est très élevée : $47 \%$ et $43 \%$. Les relations entre Miami et le Mexique sont par contre très faibles avec moins de $1 \%$. Ceci s'explique par les nombreux points de passages terrestres qui relient les deux pays. Cette situation révèle cependant le faible rôle de Mexico avec ses partenaires privilégiés que sont les pays hispanophones de l'Amérique centrale et des Caraïbes

Parmi les banques latino-américaines à Miami, les premières places sont acquises par la Lloyds TSB Bank d'Angleterre et par la Dresdnerbank Lateinamerika d'Allemagne. Les banques espagnoles occupent de bonne position Bilbao Vizcaya Argentaria (4e) et la Santander Central Hispano(10e). Toutes ont choisi Miami pour leurs affaires liées au financement du commerce extérieur entre les Etats-Unis et l'Amérique latine.

d'après Ch. Girault : Miami y las nuevas relaciones interamericanas janv. Mars 1999. Les études du CERI. Fondation Nationale des Sciences Politiques. Paris et Felipe Aldunate Montes : Yo Quiero Miami? AméricaEconomía, août 2000. Panamá.

\section{Encadré}

\section{Rio de Janeiro : opportunités d'affaires}

L'Etat de Rio de Janeiro est la porte d'entrée du Brésil et du Mercosur. Localisé dans la région la plus riche d'Amérique latine, il offre l'accès à un grand marché de plus de 200 millions de consommateurs. Deuxième état du Brésil sur le plan économique a accueilli 31,1 billon US\$ d'investissement entre 1997 et 1999. Il est prévu 28 billons $\$$ US pour 2001 qui facilitera la création de 20000 emplois.

L'Etat de Rio de Janeiro est le deuxième parc industriel au Brésil, dominé par les secteurs de la chimie, de la pétrochimie et de la pharmacie. Ces trois secteurs 
représentent plus de $15 \%$ de la production nationale. L'Etat est également très présent dans l'extraction minière et la métallurgie. Rio dirige $80 \%$ de la production du pétrole et $46 \%$ du gaz naturel provenant essentiellement du bassin de Campos. Outre Petrobras, la plus grande compagnie nationale, Rio abrite la Société de Sidérurgie nationale (CSN) acier plat, Cosigua acier non plat et Valesul (aluminium). La ville de Rio accueille les sièges sociaux nationaux ou continentaux dequelques unes des multinationales, tel Xerox, Amoco, Shell, Texaco, Esso, Coca-Cola

Combinant le développement économique avec la qualité de la vie, Rio de Janeiro a été classé comme la meilleure option pour les investissements au Brésil. Andersen Consulting 2000 auprès de 1200 investisseurs.

D'après GODIN, Company for industrial development of the state of Rio de Janeiro. Rio 2000. 